\title{
A Central Role for Heme Oxygenase-1 in the Control of Intestinal Epithelial Chemokine Expression
}

\author{
Joseph C. Onyiah Rachel E.M. Schaefer Sean P. Colgan \\ Department of Medicine, Denver Veterans Administration Medical Center and the Mucosal Inflammation Program, \\ University of Colorado School of Medicine, Aurora, CO, USA
}

\section{Keywords}

Intestinal epithelium · Cytokines · Chemokines .

Inflammation · Homeostasis · Colitis

\begin{abstract}
In mucosal inflammatory disorders, the protective influence of heme oxygenase-1 (HO-1) and its metabolic byproducts, carbon monoxide (CO) and biliverdin, is a topic of significant interest. Mechanisms under investigation include the regulation of macrophage function and mucosal cytokine expression. While there is an increasing recognition of the importance of epithelial-derived factors in the maintenance of intestinal mucosal homeostasis, the contribution of intestinal epithelial cell (IEC) HO-1 on inflammatory responses has not previously been investigated. We examined the influence of modulating HO-1 expression on the inflammatory response of human IECs. Engineered deficiency of HO-1 in Caco-2 and T84 IECs led to increased proinflammatory chemokine expression in response to pathogenic bacteria and inflammatory cytokine stimulation. Crosstalk with activated leukocytes also led to increased chemokine expression in HO-1-deficient cells in an IL-1 $\beta$ dependent manner. Treatment of Caco- 2 cells with a pharmacological inducer of $\mathrm{HO}-1$
\end{abstract}

๑) 2018 S. Karger AG, Basel led to the inhibition of chemokine expression. Mechanistic studies suggest that $\mathrm{HO}-1$ and HO-1-related transcription factors, but not HO-1 metabolic products, are partly responsible for the influence of HO-1 on chemokine expression. In conclusion, our data identify $\mathrm{HO}-1$ as a central regulator of IEC chemokine expression that may contribute to homeostasis in the intestinal mucosa.

(c) 2018 S. Karger AG, Basel

\section{Background}

Epithelial cells are unique regulators of the mucosal immune response, related to their function of serving as the barrier between the external luminal environment and the mucosal immune system. Crosstalk between the epithelium and the immune system is dictated by a complex interplay of host genetic factors, the luminal microbiota, and environmental contributions. Despite exposure to high concentrations of various potentially inflammatory environmental and bacterial antigens on their apical surface, intestinal epithelial cells (IECs) contribute to the immune tolerant state of the intestinal mucosa through dampened homeostatic responses, in the ab- 
sence of barrier disruption. IECs additionally have the complicated role of regulating the uptake of nutritionally derived macromolecules from the intestinal lumen. One of these molecules is heme, which is preferentially absorbed by the proximal small intestine. Heme is metabolized by the heme oxygenase (HO) group of enzymes into biliverdin, carbon monoxide (CO), and ferrous iron. $\mathrm{HO}-1$ is the inducible isoform of $\mathrm{HO}$, which is present in most tissues of the body but is highly expressed in the liver, spleen, and intestines [1]. HO-1 and its metabolites have been demonstrated to have anti-inflammatory, antiapoptotic, and antioxidative properties [2, 3]. HO-1 induction and $\mathrm{CO}$ exposure are protective in multiple murine models of inflammatory disease, including models of experimental colitis, through the regulation of pro- and anti-inflammatory cytokine expression, and the promotion of the macrophage antibacterial function [4-7].

While an anti-inflammatory role has been ascribed to immune cell HO-1/CO signaling, the influence of HO-1 in IECs has not been well explored. There is an increased appreciation of the role of epithelial-derived factors in mucosal immunity [8]. While the epithelium may not be the major source of cytokines produced in the intestinal mucosa, it is a rich source of various homeostatic cytokines. For example, epithelial-derived IL-8 and TGF- $\beta$ are critically important for recruiting the resident mucosal macrophage population [9]. The epithelium also plays a critical role in the response to pro- and anti-inflammatory cytokine signaling [8]. Disruption of the epithelial barrier results in bacterial invasion and an organized innate immune response, including the elaboration of several chemoattractants from the epithelium. Several epithelial-derived chemokines are implicated in the pathogenesis of inflammatory bowel disease [10-13]. Recruitment to and trafficking of neutrophils across the epithelium is a consistent feature in conditions like human ulcerative colitis, where findings such as crypt abscesses are a common manifestation.

We have previously shown that transmigration of neutrophils across epithelial cells molds epithelial gene expression, most prominently through localized oxygen depletion and the stabilization of hypoxia-inducible factor (HIF) [14]. This signaling promotes protective/restorative epithelial responses in the setting of inflammation [15]. One of the genes upregulated in this setting is the HO-1 gene, HMOX1. Here, we evaluate the influence of epithelial HO-1 on immune responses from IECs. Our findings suggest that $\mathrm{HO}-1$ contributes a regulatory check on epithelial inflammatory responses and may contribute to maintaining a homeostatic balance in the mucosa.

\section{Methods}

\section{Reagents}

Cobalt(III) protoporphyrin IX chloride (CoPP) was from Frontier Scientific (Logan, Utah). Human TNF and IL-1 $\beta$ cytokines were from Invitrogen (Carlsbad, CA, USA). The Bacterial strain used was Salmonella enterica serovar Typhimurium from the American Type Culture Collection ([ATCC]: 700408; Manassas, VA, USA). Actinomycin D, tricarbonyldichlororuthenium (II) dimer (CORM-2) and its negative control ruthenium (II) chloride hydrate $(\mathrm{RuCl} 3)$ were obtained from Sigma-Aldrich (St. Louis, MO, USA). An inhibitor of the p38 mitogen-activated protein kinase, SB202190, was obtained from Santa Cruz Biotechnology (Dallas, TX, USA). Compounds were tested for cytotoxicity using the cell counting kit-8 (Sigma-Aldrich), with all compounds used demonstrating $>90 \%$ viability within the experimental parameters compared to untreated cells.

\section{Cell Culture}

Human Caco-2 and T84 IECs as well as the monocytic THP-1 cell line were obtained from the ATCC and cultured according to standard protocol in $95 \%$ air with $5 \% \mathrm{CO}_{2}$ at $37^{\circ} \mathrm{C}$ [16]. Lentiviral transduction of Sigma MISSION ${ }^{\circledR}$ shRNA against HMOX1 (TRCN0000290436) and a non-targeting control (NTC) shRNA (SHC216) was performed using established protocols (University of Colorado Functional Genomics Facility, Aurora, CO, USA) [17]. THP-1 cells were exposed to bacteria at a MOI of 10:1. IL-1 receptor antagonist (Anakinra, Amgen, Thousand Oaks, CA, USA) was used at a concentration of $100 \mathrm{ng} / \mathrm{mL}$. Polarized IECs were grown on Transwell inserts with a $0.4-\mu \mathrm{m}$ pore size (Corning, Tewksbury, MA, USA), and cultured at least overnight with THP1 cells for co-culture experiments.

\section{Western Blot}

Western immunoblot analyses were performed on whole-cell extracts from Caco- 2 cells in RIPA buffer. Blots were incubated overnight with anti-HO-1 (1:1,000; ADI-SPA-895, ENZO, Farmingdale, NY, USA) and anti- $\beta$-actin (ab8227; Abcam, Cambridge, MA, USA). Immunoreactive proteins were visualized using Gel-Doc imager with Image Lab ${ }^{\mathrm{TM}}$ software (Bio-Rad, Hercules, CA, USA).

\section{Cytokine Immunoassay}

Human IL-8 immunoassay (BioLegend, San Diego, CA, USA) was used according to the manufacturer's instructions. A human IL- $1 \beta$ immunoassay (MSD Diagnostics, Rockville, MD, USA) was performed according to the manufacturer's instructions. Human NRF2 colorimetric cell-based ELISA (Assay Biotechnology Co., Fremont, CA, USA) was performed according to the manufacturer's instructions. Results were normalized to cell density as determined by crystal violet staining, and confirmed with GAPDH as an internal positive control. The activation of human p38 MAPK was assessed using cell-based ELISA to measure levels of phosphorylated (phospho)-Thr180 and phospho-Tyr182 compared to total p38 MAPK (antibodies \#9211 and \#9212, Cell Signaling Technology, Danvers, MA, USA).

\section{Luciferase Assay}

Promoter constructs for phospho-NF- $\kappa \mathrm{B}$-luciferase and phospho-AP-1 have been described previously [18]. Transfection of 
Caco-2 cells was performed using Lipofectamine ${ }^{\mathrm{TM}}$ LTX with PLUS $^{\text {TM }}$ reagent (ThermoFisher Scientific, Waltham, MA, USA). Following overnight transfection, cells were assayed for luciferase activity at $6 \mathrm{~h}$ after stimulation, respectively, with IL- $1 \beta$ at $1 \mathrm{ng} / \mathrm{mL}$ using the luciferase reporter assay system on a GloMax-multi microplate multimode reader (both from Promega, Madison, WI, USA). Luciferase signal was controlled using total protein via Bradford assay or a control Renilla luciferase-expressing promoter.

\section{Quantitative Polymerase Chain Reaction}

RNA was extracted using TRIzol reagent (Invitrogen), and first-strand complementary DNA synthesis was performed with $1 \mu \mathrm{g}$ of total RNA using iScript reverse transcription supermix (Bio-Rad). Real-time qPCR was performed using SYBR green on an ABI 7900HT fast real-time PCR system (both from Applied Biosystems, Carlsbad, CA, USA). $\beta$-Actin (ACTB) was used as the housekeeping gene. Primer sequences were: $\mathrm{ACTB}$, sense $5^{\prime}$ CACTCTTCCAGCCTTCCTTCC-3', antisense 5'-CAGGTCTTTGCGGATGTCCACG-3'; HMOX1, sense 5'-CATGACACCAAGGACCAGA-3' ${ }^{\prime}$, antisense $5^{\prime}$-AGTGTAAGGACCCATCGGAG-3'; IL-8, sense 5'-CTGGCCGTGGCTCTCTTG-3', antisense $5^{\prime}$-CCTTGGCAAAACTGCACCTT-3'; MIP3a (CCL20), sense $5^{\prime}$-CTGGCTGCTTTGATGTCAGT-3', antisense $5^{\prime}$-CGTGTGAAGCCCACAATAAA-3'; CXCL1, sense 5'-AACCGAAGTCATAGCCACAC- $3^{\prime}$, antisense $5^{\prime}$-GTTGGATTTGTCACTGTTCAGC-3'; MCP1, sense 5' ${ }^{\prime}$-ATCAATGCCCCAGTCACC-3', antisense $5^{\prime}$-AGTCTTCGGAGTTTGGG-3'.

\section{Statistical Analysis}

Unless otherwise indicated, values are given as means +/- standard error of the mean. Statistical analysis was performed using GraphPad Prism v7 for Windows (San Diego, CA, USA). Differences between groups were evaluated using the unpaired Student $t$ test or one-way analysis of variance with the Fisher LSD where appropriate. Two-tailed $p<0.05$ was considered to be significant.

\section{Results}

\section{HO-1 Deficiency Enhances Chemokine Release from} Human IECs

During active mucosal inflammation, the epithelium coordinates site-specific recruitment of leukocytes to the intestinal mucosa through the elaboration of chemokines [19]. This is often augmented by signaling from resident immune cells like macrophages and their proinflammatory cytokines such as IL- $1 \beta$ and TNF [8]. To determine whether HO-1 influences IEC inflammatory responses, $\mathrm{HO}-1$ was targeted for knockdown in Caco-2 cells (HO-1 $\mathrm{KD})$ using lentiviral-transduced vectors expressing HMOX1 shRNA, with a corresponding NTC shRNA vector-expressing line serving as the control. A significant reduction in HMOX1 mRNA and protein was determined by qPCR (online suppl. Fig 1A; for all online suppl. material, see www.karger.com/doi/10.1159/000488914) and
ELISA (Fig. 1a) compared to the NTC shRNA-transduced cells. Knockdown of HO-1 was also achieved using the same methods in T84 epithelial cells (online suppl. Fig. 1B), with comparable success.

To define the contribution of IEC HO-1 to stimulated chemokine expression, we modeled inflammation by exposing IECs to recombinant IL- $1 \beta$, TNF $\alpha$, or both. The expression of various epithelial chemokines was assessed by $q$ PCR and ELISA. TNF $\alpha$, and especially IL- $1 \beta$, induced chemokine expression in both Caco- 2 and T84 cells. Targeted knockdown of HO-1 resulted in a significant increase in cytokine-induced expression of the chemokines IL-8 (CXCL8, Fig. 1b-d), CCL20 (MIP3A, Fig. 1e), CXCL1 (Fig. 1f), and MCP-1 (CCL2, Fig. 1g). Indeed, the loss of HO-1 enhanced mRNA expression of IL-8 by as much as 5 times more than similarly stimulated NTC shRNA cells (Fig. 1c, $p<0.001$ ). To a lesser extent than with mRNA, such increases were also reflected at the protein level ( $p<0.05$, Fig. 1d).

To demonstrate that these results were not cell linespecific, similar analysis in T84 cells revealed that the loss of HO-1 resulted in significantly increased expression of IL-1 $\beta$-stimulated IL-8 (Fig. 1h) and MCP-1 (Fig. 1i) $(p<$ $0.05)$. Such findings strongly implicate the HO-1 pathway in the control of epithelial chemokine expression.

\section{Epithelial-Leukocyte Crosstalk Is Regulated by HO-1}

To extend these results and explore the influence of epithelial HO-1 on leukocyte-epithelial crosstalk, we utilized a co-culture model of IECs and monocytes/macrophages to simulate interactions that may occur in vivo. Monolayers of polarized Caco-2 cells were grown on the upper side of Transwell inserts, and co-cultured with THP-1 monocytes in the lower chamber. To activate cocultures, we infected THP- 1 cells $\left(2 \times 10^{5}\right)$ with the invasive pathogen Salmonella Typhimurium $\left(2 \times 10^{6}\right)$, and then harvested epithelial mRNA and profiled the chemokine expression (Fig. 2a, model).

Utilizing this model system, we defined whether THP1-derived IL-1 $\beta$ would stimulate epithelial chemokine expression and whether IEC HO-1 regulated such responses. As shown in Figure 2, Salmonella Typhimurium-infected THP-1 cells significantly increased the expression of IL-8, CCL20, and CXCL1 mRNA in both NTC shRNA and HO- 1 KD IECs ( $p<0.01$ for each; Fig. $2 b-d$, respectively). This response was confirmed to be primarily driven by THP-1-derived IL- $1 \beta$ since the addition of an IL-1 receptor antagonist (Anakinra) markedly diminished this chemokine response $(p<0.001$ for IL-8, CCL20, and CXCL1). We verified that, in these defined conditions, 


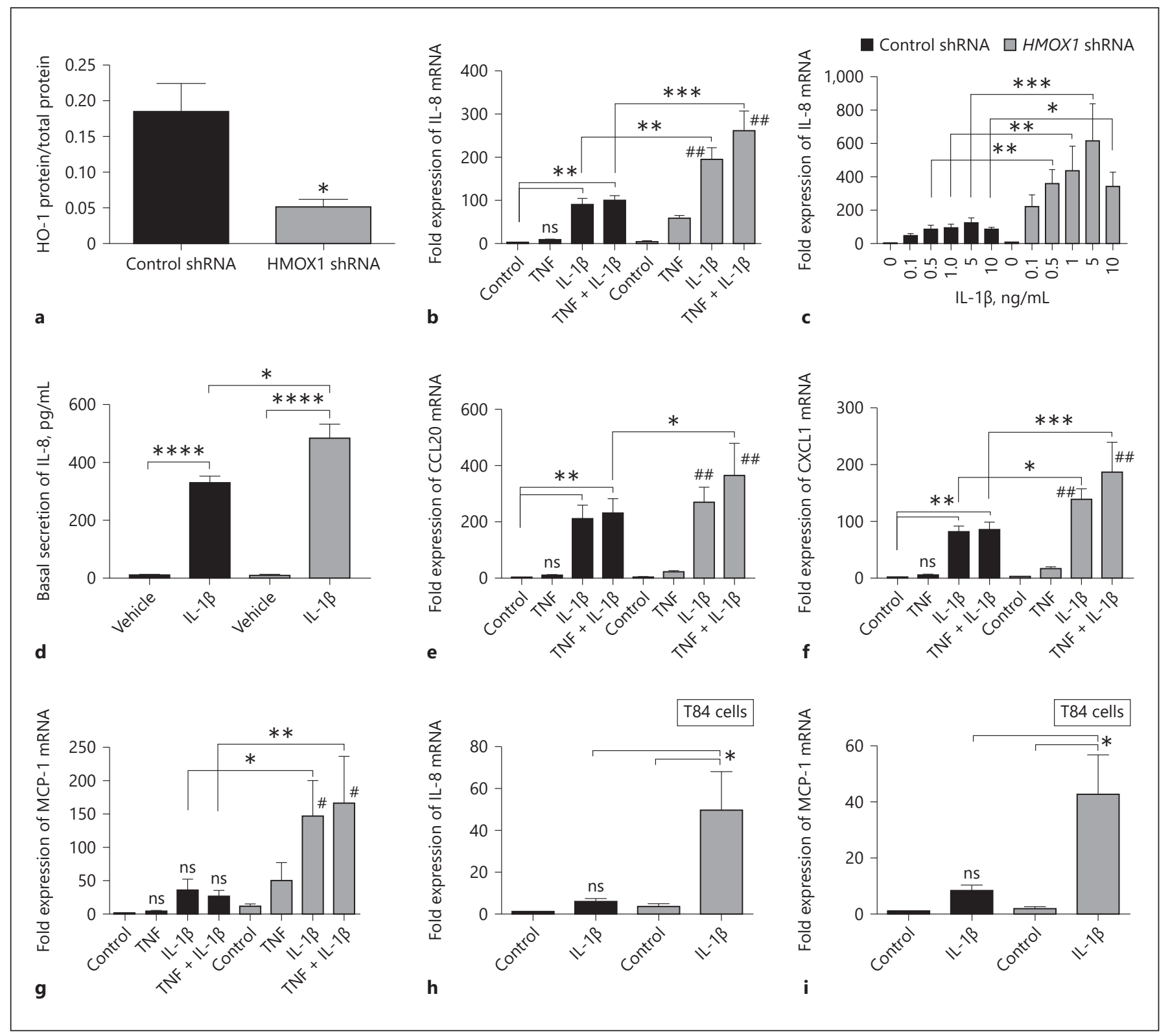

Fig. 1. HO-1 regulates chemokine expression in Caco-2 intestinal epithelial cells (IECs). a Lentiviral transduction of HMOX1 shRNA and non-targeting control shRNA in wild-type (WT) Caco-2 IECs was performed, and HO-1 protein was analyzed by ELISA. Expression of chemokines by confluent monolayers $4 \mathrm{~h}$ after stimulation with IL- $1 \beta$ ( $10 \mathrm{ng} / \mathrm{mL}$, unless otherwise stated) and/or TNF was compared to vehicle (Control) in HMOX1 shRNA- and control shRNA-transduced Caco-2 IECs as measured

Salmonella Typhimurium induced the prominent expression and secretion of IL- $1 \beta$ from THP-1 cells (Fig. 2e; $p<0.05)$, and that IL- $1 \beta$ secretion was consistent when exposed to NTC shRNA and HO-1 KD cells (Fig. 2f). by qPCR and ELISA for IL-8 (b-d), CCL20 (e), CXCL1 (f), and MCP-1 (g). IL-1 1 -induced $(5 \mathrm{ng} / \mathrm{mL})$ expression of IL-8 (h) and MCP-1 (i) was also compared to vehicle in T84 HMOX1 shRNA and control shRNA IECs. Data represent the combined results from at least 3 independent experiments. ${ }^{*} p<0.05$; ${ }^{* *} p<0.01$; ${ }^{* * *} p<0.001 ; * * * * p<0.0001$ compared to untreated control or other comparisons shown on graph. ${ }^{\#} p<0.01$ and ${ }^{\# \#} p<0.0001$ versus untreated control HMOX1 shRNA group.

To evaluate the influence of epithelial HO-1 on responses to luminal bacteria, we exposed the apical surface of the polarized IECs to a gram-negative enteric pathogenic bacterium (Salmonella Typhimurium) in the pres- 
ence and absence of THP-1 cells (Fig. 2g). After $6 \mathrm{~h}$ of exposure, we evaluated the expression of IL- 8 from our control and from HO-1 KD IECs using qPCR. Salmonella Typhimurium elicited an increase in IL-8 expression that was enhanced in the HO-1-deficient cells (Fig. 2h). This was significantly increased in the presence of THP-1 cells $(p<0.01)$. Previous work demonstrated that crosstalk between IECs and leukocytes can promote an inflammatory response to bacterial exposure on IEC apical membranes [20]. These results suggest that $\mathrm{HO}-1$, in part, also regulates the magnitude of epithelial inflammatory responses to bacterial challenge.

Consistent with our findings of direct stimulation with recombinant IL-1 $\beta$ (Fig. 1), the knockdown of IEC HO-1 enhanced IL-8 $(p<0.0001)$, CCL20 $(p<0.0001)$, and CXCL1 $(p<0.05)$ mRNA responses to activated THP-1 cells and pathogenic bacteria $(p<0.01)$, indicating that even in a more complex and physiologically relevant setting, the HO-1 pathway is central to the regulated IEC expression of chemokines.

\section{Induction of HO-1 Inhibits IEC Chemokine Release}

We next sought to determine if, alternatively, increasing HO- 1 could impact IEC chemokine expression. To do this, endogenous HO-1 was induced in the wild-type (WT) Caco-2 cell line using CoPP, a potent heme analog small-molecule inducer of HO-1 expression. Treatment with CoPP resulted in a significant increase in HO-1 mRNA (Fig. 3a; $p<0.01$ ) and protein (Fig. 3b) expression. Compared to vehicle controls, CoPP-pretreated cells, subsequently treated with IL- $1 \beta$, demonstrated diminished induction of the chemokines IL-8 and CCL20, but not of CXCL1 (Fig. 3c, d).

\section{HO-1 Metabolic Products Do Not Suppress IL-8}

\section{Expression}

Consequences of HO-1 induction are frequently mediated by HO- 1 metabolic products such as $\mathrm{CO}$ and biliverdin, which is further metabolized to bilirubin. Caco-2 IECs have been demonstrated to take up and metabolize exogenous HO-1 metabolic products [21-23]. To determine if these molecules regulate chemokine expression, we treated our control and HO-1-deficient IECs overnight with biliverdin and its product bilirubin (Fig. 4a, b). Neither biliverdin nor bilirubin significantly impacted IL- $1 \beta$-induced IL- 8 expression, and there was no significant change in the relative IL- 8 expression in the HO1-deficient cells compared to in controls. Alternatively, exogenous $\mathrm{CO}$ has been demonstrated to have an inhibitory influence on chemokine expression in IECs stimu-

HO-1 Regulates Epithelial Inflammatory

Responses lated with a mixture of cytokines (IL- $1 \beta$, TNF, and IFN- $\gamma$ ) [21]. We exposed our Caco-2 cells briefly to CO via a COreleasing molecule (CORM-2 at $100 \mu \mathrm{M})$ as previously described, and again examined the IL- 8 release after stimulation with IL-1 $\beta$. Contrary to previous studies, we did not see a significant decrease in IL-8 secretion. Instead, we saw a small increase, suggesting a more complicated mechanism of regulation of IL- 8 in response to IL- $1 \beta$ alone compared to a mixture of cytokines (Fig. 4c).

\section{Mechanisms of Regulation of Epithelial Chemokines}

by $\mathrm{HO}-1$

To determine the role of transcriptional regulation of chemokine expression by HO-1, actinomycin D (ActD) was used to block transcription in IL- $1 \beta$-treated Caco-2 cells. After $1 \mathrm{~h}$ of IL-1 $\beta$ exposure, significantly higher levels of IL- 8 were expressed in the HO- 1 KD cells compared to in the NTC shRNA cells. The addition of ActD after $1 \mathrm{~h}$ subsequently resulted in a marked and similar reduction in IL-8 expression in both controls and HO-1 KD cells at $2-4 \mathrm{~h}$ (Fig. 5c), indicating that the enhanced IL-8 expression in HO-1 KD IECs is secondary to increased transcriptional activation but not stabilization of the mRNA.

The transcription factor, nuclear factor erythroid 2-related factor 2 (NRF2) is activated under cellular stress and inflammation, and is largely responsible for the activation of HMOX1 transcription in mice and humans [24]. NRF2 has also been shown to promote IL-8 transcription in certain cells $[25,26]$. A likely compensatory increase in NRF2 in the setting of HO-1 deficiency was felt to potentially play a role in IL-8 expression. Therefore, we measured NRF2 protein in our control and HO-1 KD Caco-2 IECs by indirect ELISA (Fig. 5b). We found significantly increased NRF2 protein in our HO-1 KD cells.

Previous studies have shown that the transcription factors AP- 1 and NF- $\kappa B$ are critical to the activation of IL- 8 transcription by IL- $1 \beta[27,28]$. Therefore, we examined the relationship between HO-1 expression and activity of these transcription factors using luciferase reporter constructs. Caco-2 cells were transiently transfected with AP-1 and NF- $\kappa$ B reporter plasmids, and treated with IL$1 \beta(1 \mathrm{ng} / \mathrm{mL}$ for $6 \mathrm{~h}$ ) (Fig. $5 \mathrm{c}, \mathrm{d})$. HO-1 deficiency increased IL- $1 \beta$-induced AP-1 $(p<0.05)$, but not NF- $\kappa$ B $(p=n s)$ activity, implicating a role for AP-1 in HO-1-dependent increases in chemokine expression.

The relationship between MAPKs, AP-1, and the induction of IL-8 transcription has been previously described [28]. In addition, p38 MAPK has been demonstrated to regulate $\mathrm{HO}-1$ via NRF2, while the inhibition 


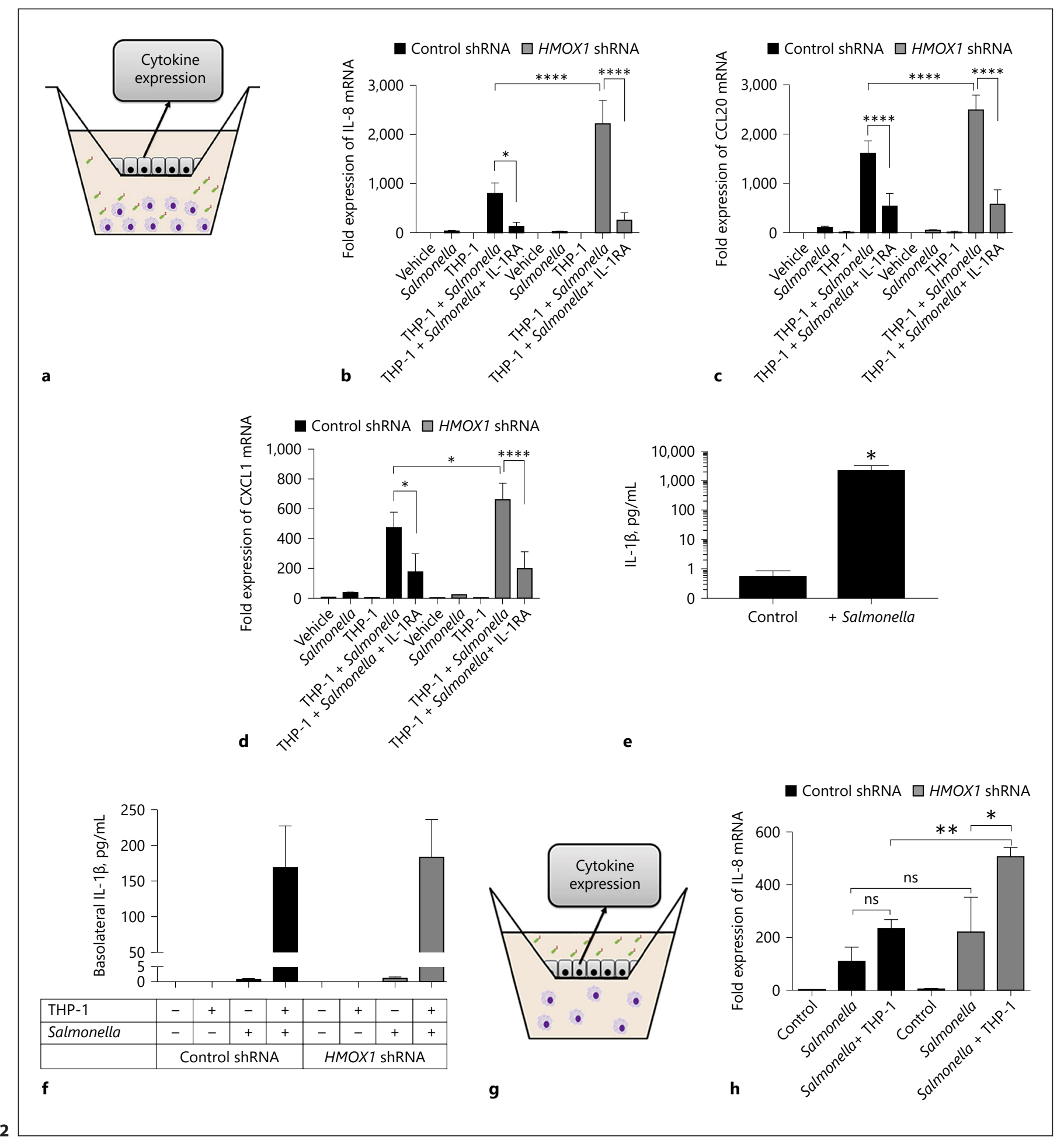

(For legend see next page.) 
of HO- 1 increases p38 MAPK activation $[29,30]$. Therefore, we assessed the level of (activated) phospho-p38 MAPK (controlled for total p38) in our HO-1-deficient cells compared to controls. We found that, after IL-1 $\beta$ treatment, there was a small but significant increase in phospho-p38 in the HO-1-deficient IECs (Fig. 5e). We then inhibited p38 MAPK to determine if it played a role in the increased IL-8 expression seen in our HO-1 KD cells in response to IL- $1 \beta$ treatment. As shown in Figure $5 f$, inhibition of p38 MAPK significantly inhibited the HO-1-dependent enhancement of IL- 8 expression at $2 \mathrm{~h}$. Such results implicate, at least in part, p38 MAPK activation in the increased chemokine induction in the setting of HO-1 deficiency.

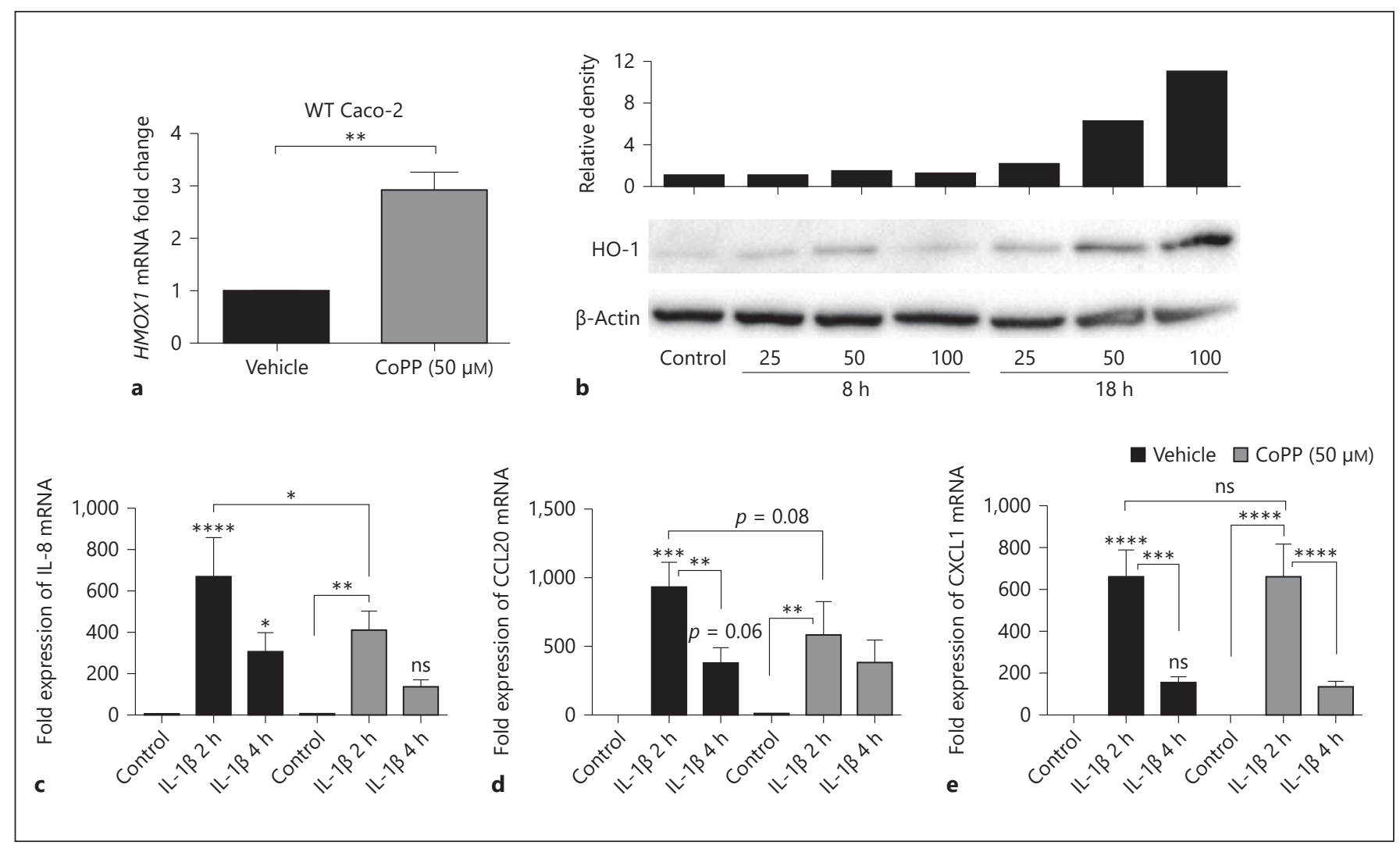

Fig. 3. HO-1 induction inhibits chemokine expression. Wild-type (WT) Caco-2 intestinal epithelial cells (IECs) were treated with 50 $\mu \mathrm{M}$ of CoPP or vehicle overnight, and HMOX1 expression was measured by qPCR (a) and Western blot (b). c-e After vehicle or CoPP treatment, WT Caco-2 cells were treated with $10 \mathrm{ng} / \mathrm{mL}$ of

Fig. 2. Epithelial HO-1 regulates leukocyte-epithelial crosstalk. a Model to examine epithelial-leukocyte interactions using polarized Caco-2 intestinal epithelial cells (IECs) in the upper chamber of a Transwell insert with THP-1 monocytes in the lower chamber, activated with Salmonella Typhimurium for $6 \mathrm{~h}$ (for mRNA) or 24 $\mathrm{h}$ (for protein). b-d Expression of IL-8, CCL20, and CXCL1 mRNA from Caco-2 IECs during co-culture with activated THP-1 cells. e IL- $1 \beta$ secretion from THP- 1 cell supernatants after $24 \mathrm{~h}$, as measured by ELISA. f IL- $1 \beta$ secretion from THP- 1 cells co-cultured with control shRNA- and HMOX1 shRNA-transduced Caco-2
IL- $1 \beta$ for 2 and $4 \mathrm{~h}$, and chemokine expression assessed by qPCR. Combined results from 3 experiments. ${ }^{*} p<0.05$; ${ }^{*} p<0.01$; *** $p<0.001$; ${ }^{* * * *} p<0.0001$ compared to untreated vehicle control or other comparisons shown on the graph.

IECs after $24 \mathrm{~h}$. Data represent combined results from at least 3 independent experiments. $g$ Model to examine physiologic bacterial-epithelial activation using polarized IECs in the upper chamber of a Transwell insert exposed to enteric bacteria in the presence (or absence) of leukocytes in the lower chamber, evaluating IEC chemokine expression. $\mathbf{h}$ Expression of IL-8 mRNA from Caco-2 IECs $6 \mathrm{~h}$ after exposure of the apical surface to pathogenic bacteria in the presence or absence of THP-1 monocytes. Combined results from 3 independent experiments. ${ }^{*} p<0.05$; ${ }^{* * *} p<0.001$; **** $p<0.0001$. 


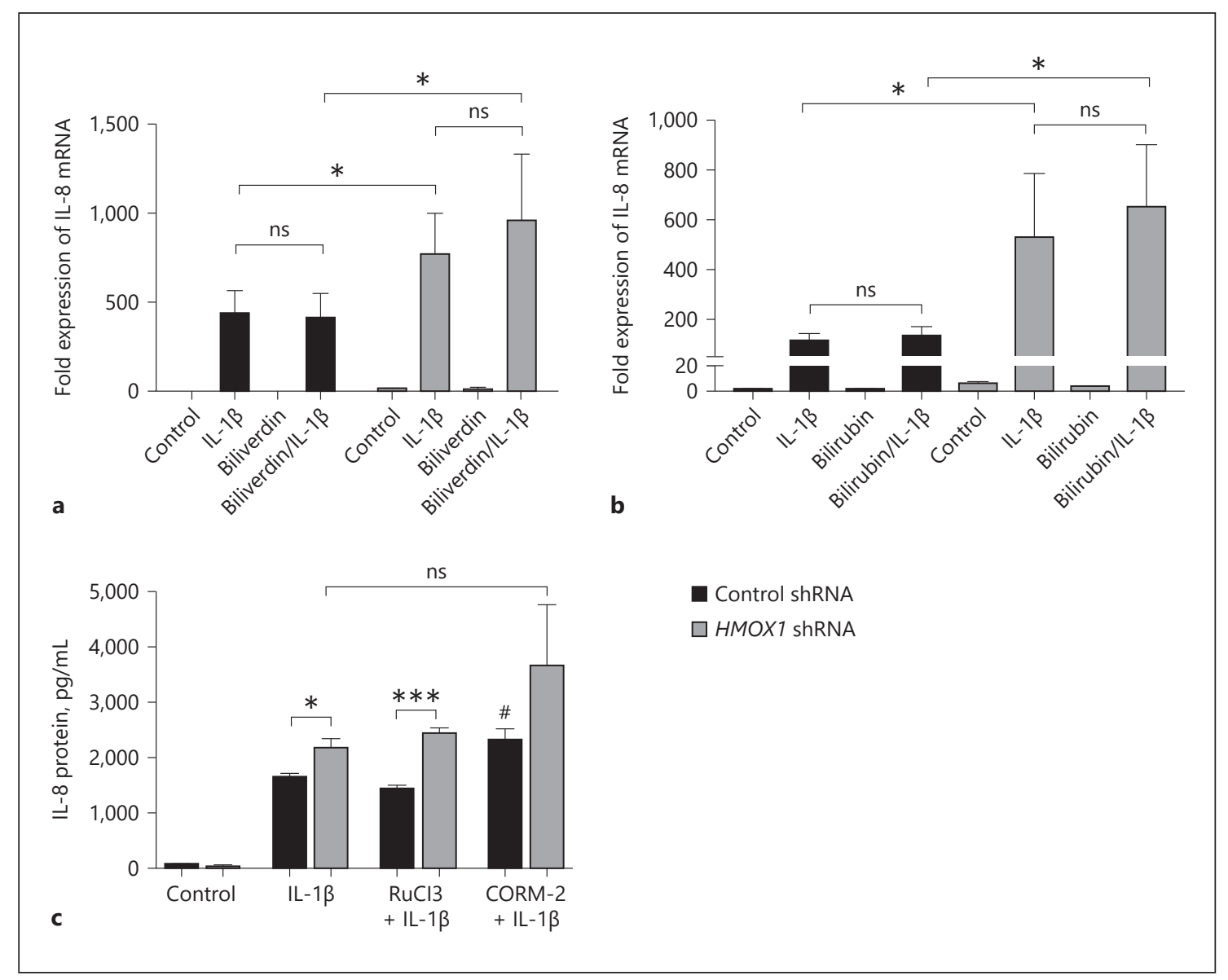

Fig. 4. The role of HO-1 metabolic products. a, b Control and HO1-deficient Caco-2 intestinal epithelial cells (IECs) were treated with biliverdin and bilirubin $(50 \mu \mathrm{M})$ overnight and then stimulated with IL-1 $\beta(1 \mathrm{ng} / \mathrm{mL})$ for $3 \mathrm{~h}$. IL- 8 was measured by qPCR. Data reflect combined results from at least 3 independent experiments. c Cells were exposed to a freshly prepared CO-releasing molecule CORM-2 for $1 \mathrm{~h}$, or to its negative control $\mathrm{RuCl} 3$, prior

\section{Discussion}

The intricate maintenance of an effective mucosal immune system in the gastrointestinal tract is composed of numerous components working in concert [31]. Induction of HO- 1 in the intestine and delivery of its metabolite, $\mathrm{CO}$, have previously been shown to contribute to the maintenance of homeostasis in various murine models of colitis [4-6]. This has been associated with augmented macrophage function, including a balance towards increased anti-inflammatory cytokine expression and decreased proinflammatory cytokine expression $[6,32]$. The role of the intestinal epithelium in the protective influence of the HO-1 pathway has not been evaluated. to stimulation with IL-1 $\beta$ ( $1 \mathrm{ng} / \mathrm{mL})$ for $24 \mathrm{~h}$. IL-8 was measured by cytokine immunoassay and normalized to milligrams of total protein. Data are representative of results from 2 independent experiments. ${ }^{*} p<0.05,{ }^{* * *} p<0.001$ versus untreated control shRNA cells or other comparisons shown. ${ }^{*} p<0.05$ versus IL-1 $\beta$-treated control shRNA cells.

The increasingly understood importance of epithelialderived factors and responses has expanded our view of the epithelium as an important contributor to the immune response and as a regulator of mucosal homeostasis $[8,9,33]$. Dysregulated expression of epithelial-derived chemokines has been strongly associated with the pathogenesis of inflammatory bowel disease (IBD) $[10,12,34$, 35]. The result of increased chemokine expression in the intestine typically includes the increased recruitment and activation of immune cells and increased proinflammatory cytokine expression in the mucosa [8], and can lead to a loss of epithelial barrier integrity, increased bacterial invasion, and perpetuation of inflammation [33]. Our results identify a prominent role for $\mathrm{HO}-1$ in the regulation 


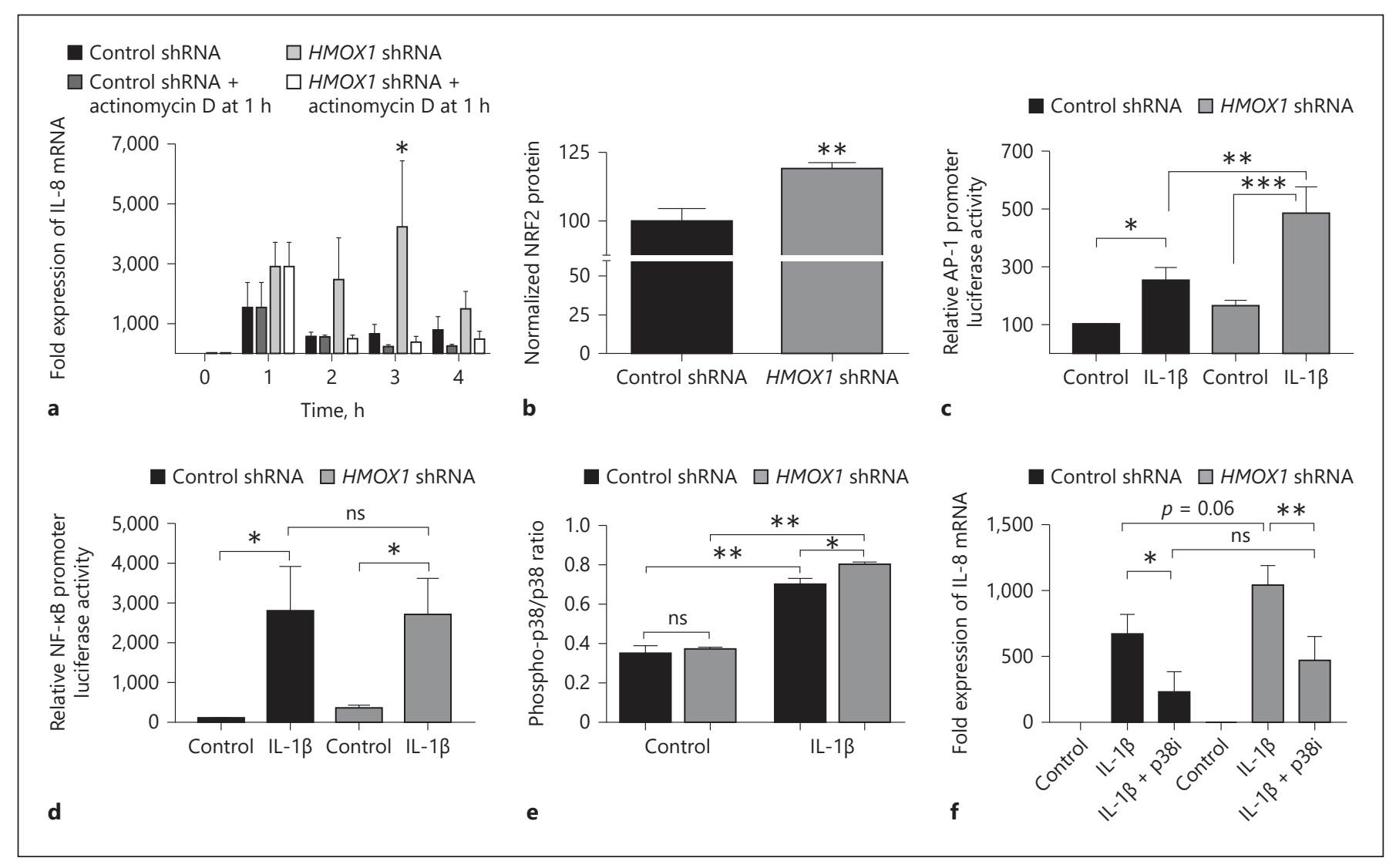

Fig. 5. Mechanisms of transcriptional regulation by HO-1. a Expression of IL-8 in confluent control shRNA- and HMOX1 shRNA-transduced Caco-2 IECs treated with IL-1 $\beta$ ( $1 \mathrm{ng} / \mathrm{mL})+/-$ transcriptional blockade with actinomycin D after $1 \mathrm{~h}$. b Expression of NRF2 protein at baseline normalized to cell density via crystal violet staining and presented as $\%$ of control. The activation of AP-1 (c) and NF- $\kappa B(\mathbf{d})$ transcription factors by IL- $1 \beta(1 \mathrm{ng} / \mathrm{mL})$ was measured using luciferase reporter plasmid transfection in non-targeted control shRNA- and HMOX1 shRNA-transduced
Caco-2 IECs. e Activation (phosphorylation) of p38 MAPK was assessed at baseline and in the setting of IL- $1 \beta$ treatment $(1 \mathrm{ng} / \mathrm{mL}$ $\times 30 \mathrm{~min}$ ) by cell-based ELISA. $\mathbf{f}$ The influence of inhibition of p38 MAPK on the increased expression of IL- 8 in HMOX1 shRNA Caco- 2 cells was assessed by pretreatment with a p38 MAPK inhibitor (p38i) SB202190 at $40 \mu \mathrm{M}, 1 \mathrm{~h}$ prior to IL-1 $\beta(1 \mathrm{ng} / \mathrm{mL})$ exposure for $2 \mathrm{~h}$. Data represent the combined results from at least 3 independent experiments. ${ }^{*} p<0.05$; $^{* *} p<0.01$. of epithelial chemokine expression. Using HO-1 loss-/ gain-of-function approaches, we have found that the epithelial HO-1 pathway regulates epithelial chemokine production and serves as an endogenous "braking" mechanism to the mucosal inflammatory response.

As part of these studies, we developed a co-culture model system to define soluble factor crosstalk between monocyte/macrophage and IECs. We previously identified the induction of epithelial HO-1 in a cohort of genes upregulated during neutrophil transepithelial migration [14]. In this setting, neutrophils induce HIF [14] and, given that HO- 1 is a well-established HIF target gene [36], it is likely that HIF contributes to epithelial HO-1 expression in this co-culture system. We developed the co-cul- ture model system to define soluble factor crosstalk between monocytes/macrophages and IECs. Consequently, we have shown that HO-1 deficiency in IECs leads to an elevated inflammatory response demonstrated by markedly increased proinflammatory chemokine expression. This appears to be the case both in the response to macrophage-derived cytokines and the exposure to pathogenic bacteria. Similar findings have recently been demonstrated for alveolar epithelial cells in a murine model of acute lung injury [37]. Diminished lipopolysaccharideinduced chemokine expression and neutrophil migration was observed after $\mathrm{HO}-1$ induction, and these were found to be dependent on non-myeloid, HO-1, including pulmonary epithelial cells and endothelial cells. 
The regulation of inflammatory responses by HO-1 has been demonstrated in other cell types as well [38]. The induction of HO-1 in astrocytes, for example, has been reported to inhibit nitric oxide production in response to IL- $1 \beta$ treatment through the modulation of p38 MAPK activation. Helicobacter pylori infection-induced secretion of IL- 8 by gastric epithelial cells also appears to be inhibited by HO-1 induction [39]. The findings of $\mathrm{HO}-1$ regulation of the IL- 8 secreted by IECs is particularly important, given the previous evidence of IECs and intestinal mast cells being the main source of basal intestinal IL-8 secretion [9]. Such basal secretion is responsible for the recruitment and protective retention of intestinal macrophages, but, in excess, can contribute to IBD pathogenesis [11]. We propose that, in this setting, HO-1 subserves the mucosal inflammatory response by functioning as an endogenous braking mechanism to uncontrolled chemokine production.

Prior studies have shown that the HO-1 metabolic byproduct CO likely plays an intermediate role in the regulation of cytokine expression in macrophages by modulating MAPKs and AP-1 $[40,41]$. In addition, exogenous $\mathrm{CO}$ (in the form of CORM-2) inhibits the inflammatory response in Caco-2 cells to a mix of inflammatory cytokines [21]. This response includes the inhibition of IL-8 expression in Caco-2 cells and the contribution of AP-1, NF- $\kappa B$, and MAPK family signaling. We have extended these findings by showing the importance of endogenous $\mathrm{HO}-1$ levels on IEC responses to pathogenic bacteria, on IEC-leukocyte signaling, and on a broader range of IEC chemokines associated with IBD. Additionally, we have shown that the HO-1 metabolic byproduct, biliverdin, which has anti-inflammatory properties in other settings, does not appear to be responsible for the regulation of IEC chemokines.

However, in contrast to previous studies, we did not see any inhibition of IL-8 expression upon CO exposure when stimulating with a single cytokine, IL- $1 \beta$, in the presence of CORM-2 in either our control or HO-1-deficient cells. Instead, we saw slightly increased IL-8 relative to controls. The influence of $\mathrm{CO}$ may be specific to the mix of cytokines used; here we used IL- $1 \beta$ only, in contrast to the previous study which stimulated cells with a combination of 3 different inflammatory cytokines [21].

Alternative mechanisms to explain our findings include a role for HO-1 protein independent of its metabolites, which has been demonstrated in other settings [4244]. In this regard, there is evidence that $\mathrm{HO}-1$ protein helps directly to coordinate the transcriptional activity of NRF2 and related oxidative stress genes. We demonstrat- ed a likely compensatory increase in NRF2 protein in our HO-1-deficient cells, which, if dysregulated, may contribute to increased chemokine (e.g., IL-8) transcription [25]. Not surprisingly, p38 MAPK and CO are both activators of NRF2, which may explain the contrasting responses we found to inhibiting p38 and CO treatment [45-47].

In conclusion, we identify $\mathrm{HO}-1$ as a central regulator of epithelial chemokine expression. Using loss-/gain-offunction approaches, we demonstrate that $\mathrm{HO}-1$, through the regulation of transcriptional mechanisms, controls the expression of multiple IEC-derived chemokines. In the absence of such control, as occurs in diseases such as IBD, mucosal inflammation may proceed in an unchecked manner. These results further suggest that administration of $\mathrm{HO}-1$-inducing agents (e.g., certain heme analogs) and/or promoting HO-1 gene expression (e.g., HIF stabilizers) may be of therapeutic benefit.

\section{Acknowledgements}

This work was supported by National Institutes of Health grants (DK103639, DK50189, and DK95491) and the US Department of Veterans Affairs (Merit Award BX002182).

\section{Disclosure Statement}

The authors declare no financial interests in any of the work submitted here.

References

1 Ryter SW, Alam J, Choi AM: Heme oxygenase-1/carbon monoxide: from basic science to therapeutic applications. Physiol Rev 2006; 86:583-650.

2 Otterbein LE, et al: Heme oxygenase-1: unleashing the protective properties of heme. Trends Immunol 2003;24:449-455.

3 Ryter SW, Choi AM: Targeting heme oxygenase-1 and carbon monoxide for therapeutic modulation of inflammation. Transl Res 2016;167:7-34.

4 Hegazi RA, et al: Carbon monoxide ameliorates chronic murine colitis through a heme oxygenase-1-dependent pathway. J Exp Med 2005;202:1703-1713.

5 Sheikh SZ, et al: An anti-inflammatory role for carbon monoxide and heme oxygenase- 1 in chronic Th2-mediated murine colitis. J Immunol 2011;186:5506-5513.

-6 Onyiah JC, et al: Carbon monoxide and heme oxygenase-1 prevent intestinal inflammation in mice by promoting bacterial clearance. Gastroenterology 2013;144:789-798.
Onyiah/Schaefer/Colgan 
7 Takagi T, et al: Inhalation of carbon monoxide ameliorates TNBS-induced colitis in mice through the inhibition of TNF- $\alpha$ expression. Dig Dis Sci 2010;55:2797-2804.

8 Onyiah JC, Colgan SP: Cytokine responses and epithelial function in the intestinal mucosa. Cell Mol Life Sci 2016;73:4203-4212.

$\checkmark 9$ Smythies LE, et al: Mucosal IL-8 and TGF- $\beta$ recruit blood monocytes: evidence for crosstalk between the lamina propria stroma and myeloid cells. J Leukoc Biol 2006;80:492-499.

10 Kwon JH, et al: Colonic epithelial cells are a major site of macrophage inflammatory protein $3 \alpha$ (MIP-3a) production in normal colon and inflammatory bowel disease. Gut 2002; 51:818-826

11 Hommes DW, et al: Production and cellular source of interleukin-8 in ulcerative colitis. Inflamm Bowel Dis 1995;1:108-116.

$\checkmark 12$ Izutani R, et al: Increased expression of interleukin-8 mRNA in ulcerative colitis and Crohn's disease mucosa and epithelial cells. Inflamm Bowel Dis 1995;1:37-47.

13 Atreya R, Neurath MF: Chemokines in inflammatory bowel diseases. Dig Dis 2010;28: 386-394.

14 Campbell EL, et al: Transmigrating neutrophils shape the mucosal microenvironment through localized oxygen depletion to influence resolution of inflammation. Immunity 2014;40:66-77.

15 Colgan SP, Campbell EL, Kominsky DJ: Hypoxia and mucosal inflammation. Ann Rev Pathol 2016;11:77-100.

16 Lanis JM, et al: Tryptophan metabolite activation of the aryl hydrocarbon receptor regulates IL-10 receptor expression on intestinal epithelia. Mucosal Immunol 2017;10:11331144.

17 Glover LE, et al: Control of creatine metabolism by HIF is an endogenous mechanism of barrier regulation in colitis. Proc Natl Acad Sci USA 2013;110:19820-19825.

18 Ehrentraut SF, et al: Perturbation of neddylation-dependent NF- $\kappa B$ responses in the intestinal epithelium drives apoptosis and inhibits resolution of mucosal inflammation. Mol Biol Cell 2016, Epub ahead of print.

-19 Szabady RL, McCormick BA: Control of neutrophil inflammation at mucosal surfaces by secreted epithelial products. Front Immunol 2013;4:220.

20 Haller D, et al: Non-pathogenic bacteria elicit a differential cytokine response by intestinal epithelial cell/leucocyte co-cultures. Gut 2000;47:79-87.
21 Megias J, Busserolles J, Alcaraz MJ: The carbon monoxide-releasing molecule CORM-2 inhibits the inflammatory response induced by cytokines in Caco- 2 cells. Br J Pharmacol 2007;150:977-986.

22 Cable JW, Cable EE, Bonkovsky HL: Induction of heme oxygenase in intestinal epithelial cells: studies in Caco-2 cell cultures. Mol Cell Biochem 1993;129:93-98.

23 Uc A, McDonagh AF, Stokes JB: Metabolism of haem in Caco-2 cells. Exp Physiol 2010;95: 296-303.

24 Alam J, et al: Nrf2, a Cap'n'Collar transcription factor, regulates induction of the heme oxygenase-1 gene. J Biol Chem 1999;274: 26071-26078.

25 Zhang X, et al: Activation of the Nrf2/antioxidant response pathway increases IL-8 expression. Eur J Immunol 2005;35:3258-3267.

26 Loboda A, et al: HIF-1 induction attenuates Nrf2-dependent IL-8 expression in human endothelial cells. Antioxid Redox Signal 2009; 11:1501-1517.

27 Campbell LM, Maxwell PJ, Waugh DJ: Rationale and means to target pro-inflammatory interleukin-8 (CXCL8) signaling in cancer. Pharmaceuticals 2013;6:929-959.

28 Hwang YS, et al: Interleukin- $1 \beta$ stimulates IL-8 expression through MAP kinase and ROS signaling in human gastric carcinoma cells. Oncogene 2004;23:6603-6611.

29 Naidu S, et al: Inhibition and genetic deficiency of p38 MAPK up-regulates heme oxygenase-1 gene expression via Nrf2. J Immunol 2009;182:7048-7057.

30 Al-Huseini LM, et al: Heme oxygenase-1 regulates dendritic cell function through modulation of p38 MAPK-CREB/ATF1 signaling. J Biol Chem 2014;289:16442-16451.

31 Abraham C, Medzhitov R: Interactions between the host innate immune system and $\mathrm{mi}-$ crobes in inflammatory bowel disease. Gastroenterology 2011;140:1729-1737.

32 Onyiah JC, et al: Heme oxygenase- 1 and carbon monoxide regulate intestinal homeostasis and mucosal immune responses to the enteric microbiota. Gut Microbes 2014;5:220224.

33 Luissint AC, Parkos CA, Nusrat A: Inflammation and the intestinal barrier: leukocyte-epithelial cell interactions, cell junction remodeling, and mucosal repair. Gastroenterology 2016;151:616-632.

34 Mitsuyama K, et al: IL-8 as an important chemoattractant for neutrophils in ulcerative colitis and Crohn's disease. Clin Exp Immunol 1994;96:432-436.
35 Puleston J, et al: A distinct subset of chemokines dominates the mucosal chemokine response in inflammatory bowel disease. Aliment Pharmacol Ther 2005;21:109-120.

-36 Lee PJ, et al: Hypoxia-inducible factor-1 mediates transcriptional activation of the heme oxygenase-1 gene in response to hypoxia. J Biol Chem 1997;272:5375-5381.

37 Konrad FM, et al: Tissue heme oxygenase-1 exerts anti-inflammatory effects on LPS-induced pulmonary inflammation. Mucosal Immunol 2016;9:98-111.

38 Sheng WS, et al: Hemin inhibits NO production by IL- $1 \beta$-stimulated human astrocytes through induction of heme oxygenase- 1 and reduction of p38 MAPK activation. J Neuroinflamm 2010;7:51.

39 Gobert AP, et al: Disruption of nitric oxide signaling by Helicobacter pylori results in enhanced inflammation by inhibition of heme oxygenase-1. J Immunol 2011;187:53705379 .

40 Morse D, et al: Suppression of inflammatory cytokine production by carbon monoxide involves the JNK pathway and AP-1. J Biol Chem 2003;278:36993-36998.

41 Otterbein LE, et al: Carbon monoxide has anti-inflammatory effects involving the mitogen-activated protein kinase pathway. Nat Med 2000;6:422-428.

$\checkmark 42$ Biswas C, et al: Nuclear heme oxygenase-1 (HO-1) modulates subcellular distribution and activation of Nrf2, impacting metabolic and anti-oxidant defenses. J Biol Chem 2014; 289:26882-26894.

43 Lin Q, et al: Heme oxygenase-1 protein localizes to the nucleus and activates transcription factors important in oxidative stress. J Biol Chem 2007;282:20621-20633.

44 Lin QS, et al: Catalytic inactive heme oxygenase-1 protein regulates its own expression in oxidative stress. Free Radic Biol Med 2008;44: 847-855.

$45 \mathrm{Ma} \mathrm{L}$, et al: p38 MAPK-dependent Nrf2 induction enhances the resistance of glioma cells against TMZ. Med Oncol 2015;32:69.

-46 Sun Z, Huang Z, Zhang DD: Phosphorylation of Nrf2 at multiple sites by MAP kinases has a limited contribution in modulating the Nrf2-dependent antioxidant response. PLoS One 2009;4:e6588.

-47 Wang B, et al: Carbon monoxide-activated Nrf2 pathway leads to protection against permanent focal cerebral ischemia. Stroke 2011; 42:2605-2610.
HO-1 Regulates Epithelial Inflammatory Responses 